\title{
Prolonged Association between Water Molecules under
}

\section{Hydrophobic Nanoconfinement}

Rui Zhang ${ }^{1}$, Diego Troya ${ }^{1 *}$, Louis A. Madsen ${ }^{1 *}$

${ }^{1}$ Department of Chemistry and Macromolecules Innovation Institute, Virginia Tech, Blacksburg, VA 24061

\section{Corresponding authors}

*Louis A. Madsen

Virginia Tech

Blacksburg, VA 24061

$\underline{\text { lmadsen@vt.edu }}$

*Diego Troya

Virginia Tech

Blacksburg, VA 24061

$\underline{\text { troya@vt.edu }}$ 


\section{Simulation models overview}
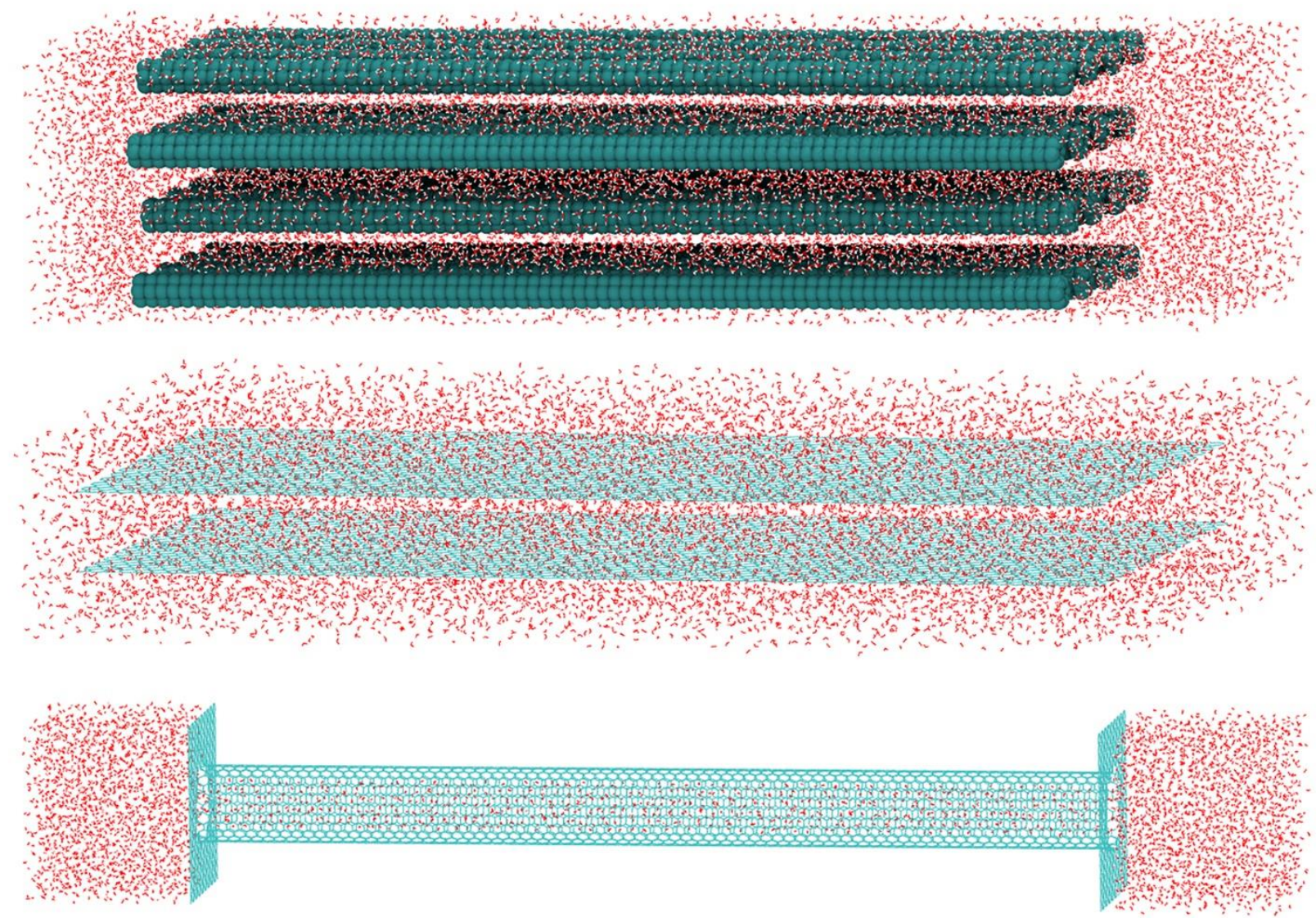

Figure S1. The complete representations of the rigid-rod model, the parallel-plate model, and the nanotube model. 


\section{Ring structure in rigid rods model}
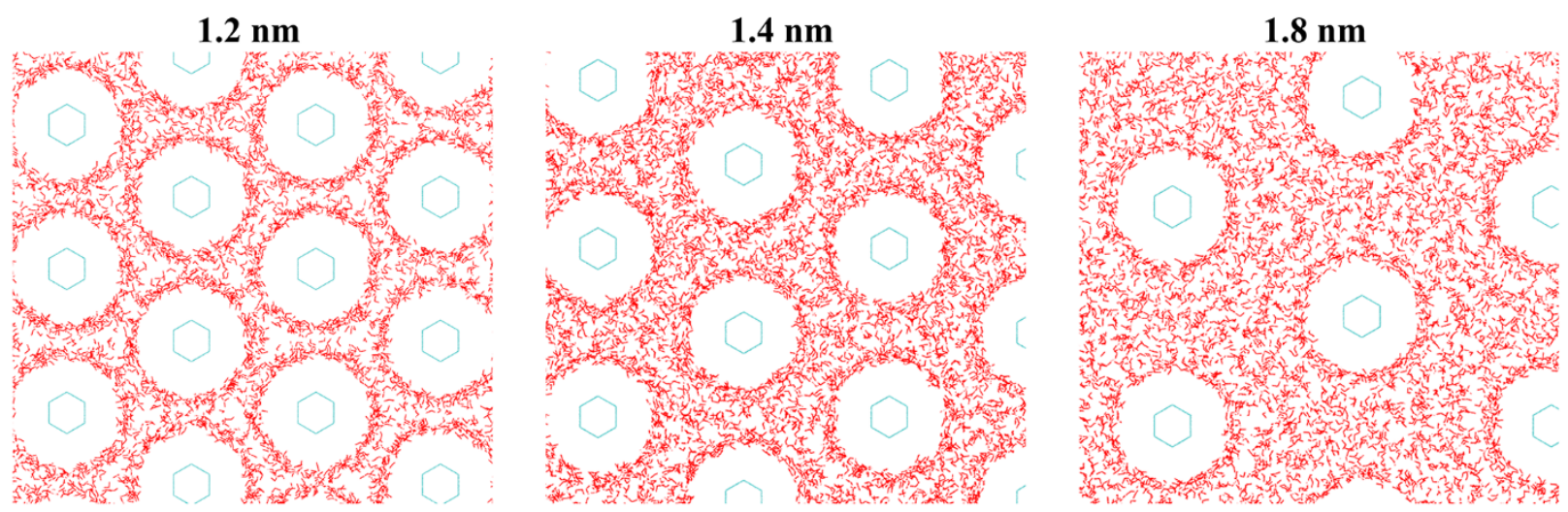

Figure S2. View of the cross-sections of the rigid-rod model of varying rod-rod distances. The first surface water layer forms a most prominent ring structure with a 1.2-nm rod-rod distance. With higher rod-rod distances, the contrast between the first surface water layer and the other water molecules becomes less distinct. 
3. Activation energy and front factor of Arrhenius plots from the rigid-rod model
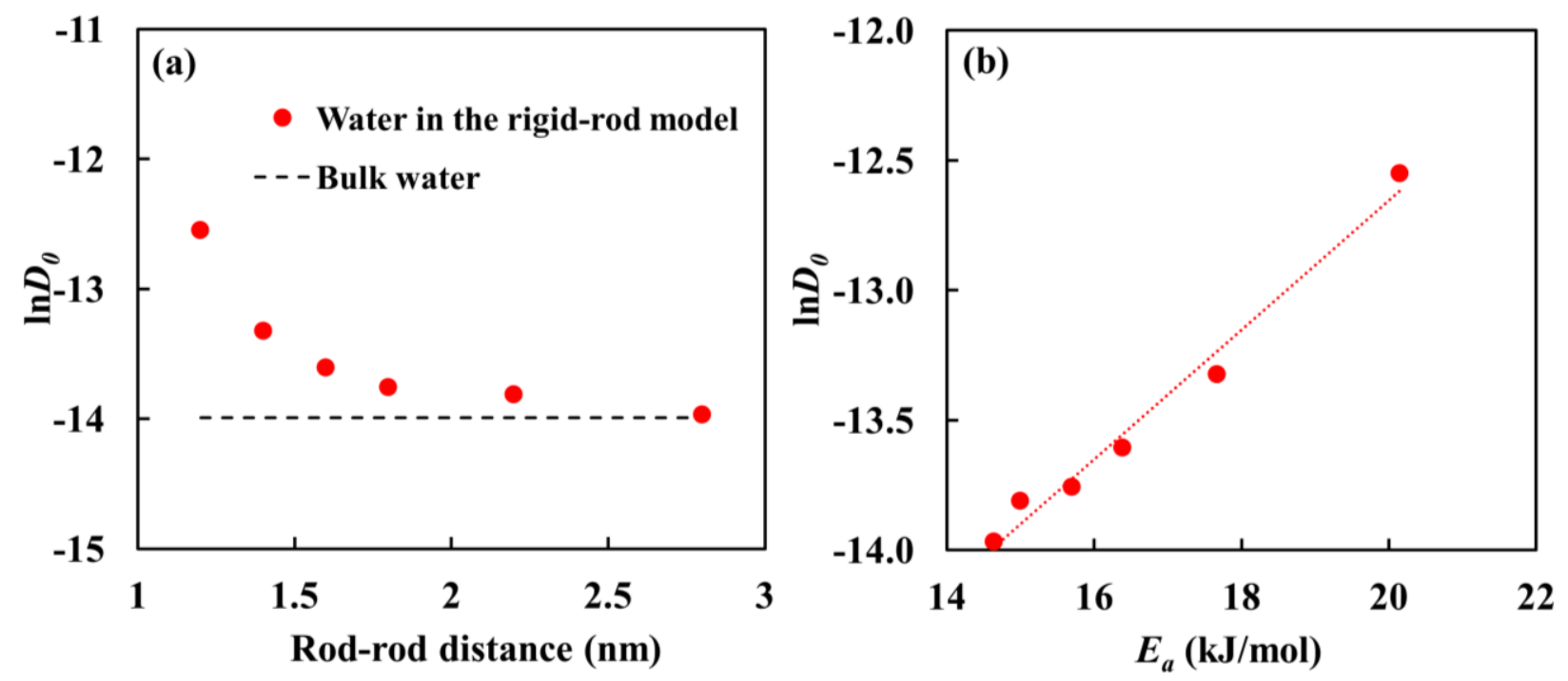

Figure S3. Insights into the Arrhenius parameters from the rigid-rod model. Panel (a) plots the front factor $\ln D_{0}$ of Arrhenius plot as a function of rod-rod distance. Panel (b) shows the compensation relation between front factor and the activation energy $E_{a}$. With decreasing confinement size, an increasing $E_{a}$ signals increasing entropy of diffusion. Meanwhile, an increasing $\ln D_{0}$ reveals that the entropy of diffusion also increases. 
4. Activation energy of diffusion as a function of confinement size in various confinement models

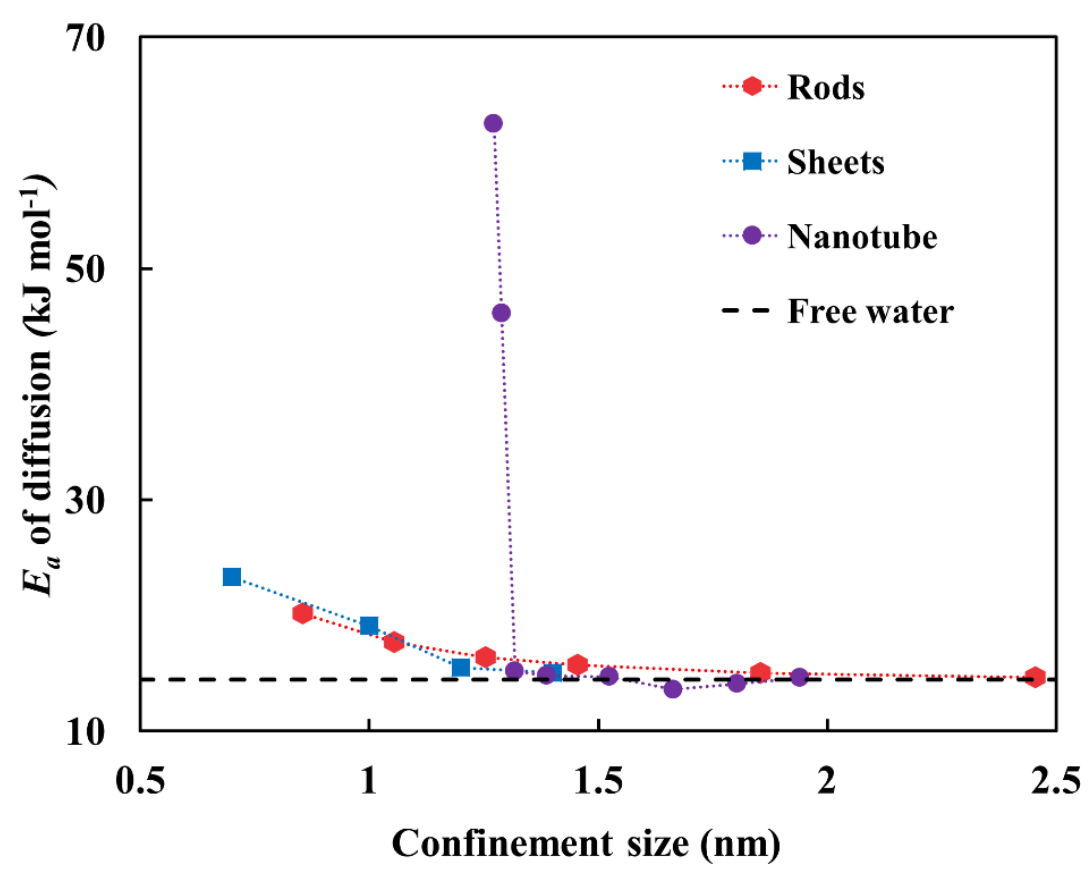

Figure S4. Plots of $E_{a}$ as a function of confinement size in the rigid-rod, parallel-plate, and CNT models. We use the distance between nearest carbons on adjacent rods, distances between the two plates, and the nanotube diameters as the confinement sizes in these models. While the $E_{a}$ in the CNT models increases rapidly with respect to decreasing confinement size, the $E_{a}$ dependence on confinement size in the rigid-rod model and the parallel-plate model follows a similar trend. In all three models, the apparent increase in $E_{a}$ starts when the confinement size is below $1.3 \mathrm{~nm}$. 


\section{The offset of reciprocal temperature in temperature-confinement superposition}
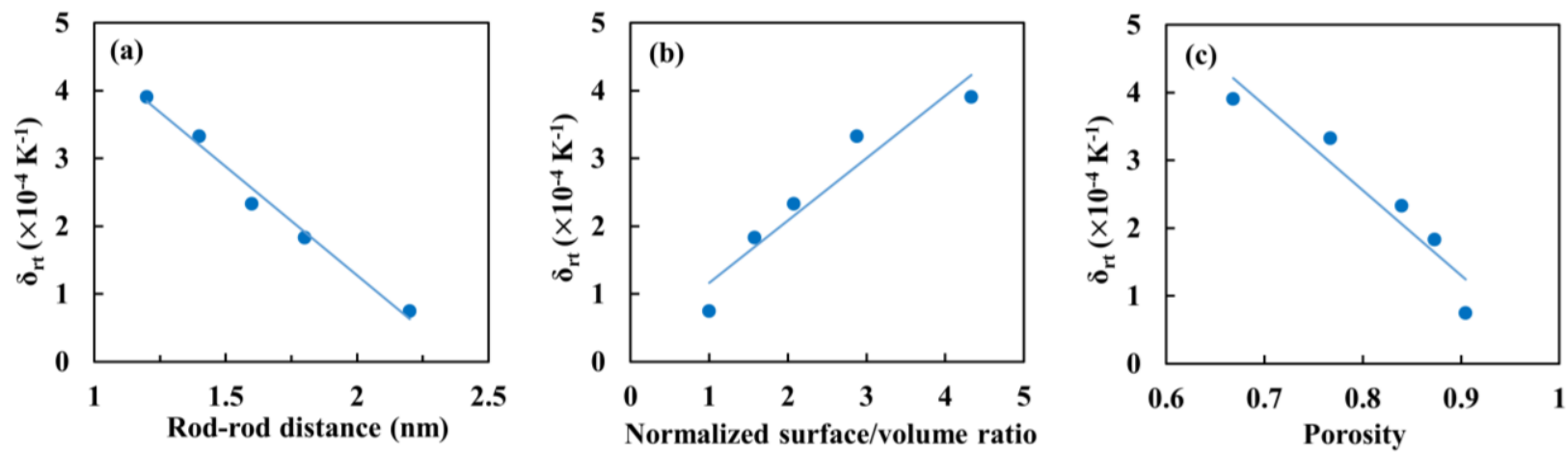

Figure S5. Plots of the reciprocal temperature offset $\left(\delta_{\mathrm{rt}}\right)$ vs. rod-rod distances (a), the normalized surface-to-volume ratio of confined water (b), and porosity of confining medium (c). To calculate the surface-to-volume ratio, we assume a constant interface area between confined water and rigid rods. We estimate the volume of confined water by assuming an effective radius of the rigid rods. ${ }^{1-}$ ${ }^{2}$ The surface-to-volume ratio at a rod-rod distance of $1.2 \mathrm{~nm}$ is normalized to 1 . 


\section{Cut-off distance consideration for residence time of water around water}
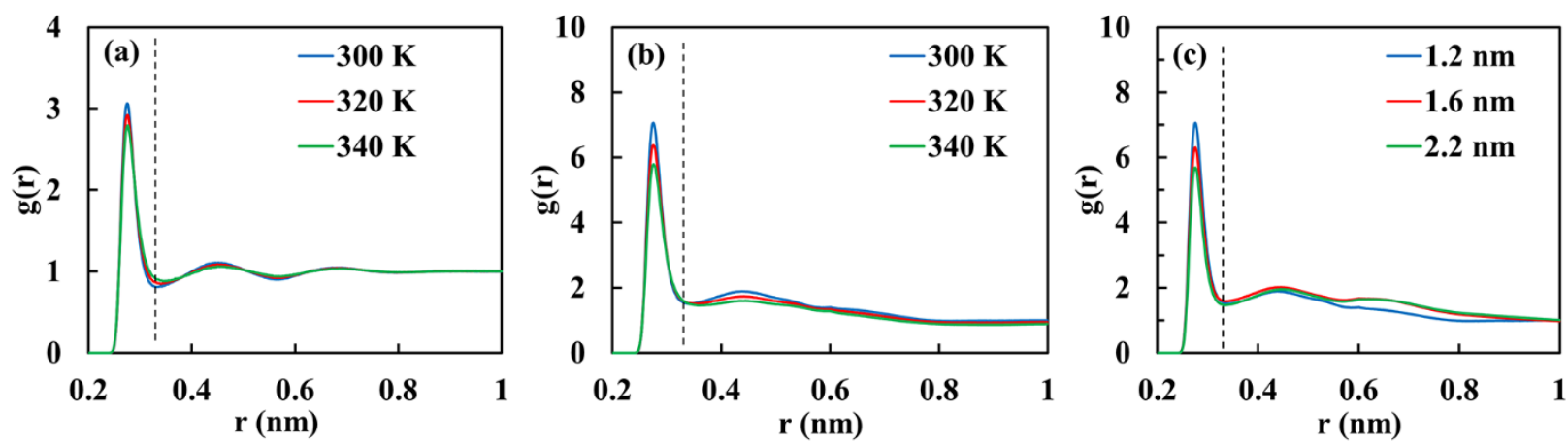

Figure S6. Radial distribution functions (RDFs) of water around water in bulk water (a), confined water in rigid rods model with a rod-rod distance of $1.2 \mathrm{~nm}(\mathrm{~b})$, and confined water at $300 \mathrm{~K}$ in rigid rods model (c). The dashed line represents the cut-off distance $0.33 \mathrm{~nm}$. We select the cutoff distance based on the first minimal in RDFs. This cut-off distance works reasonably well with both confined and bulk water.
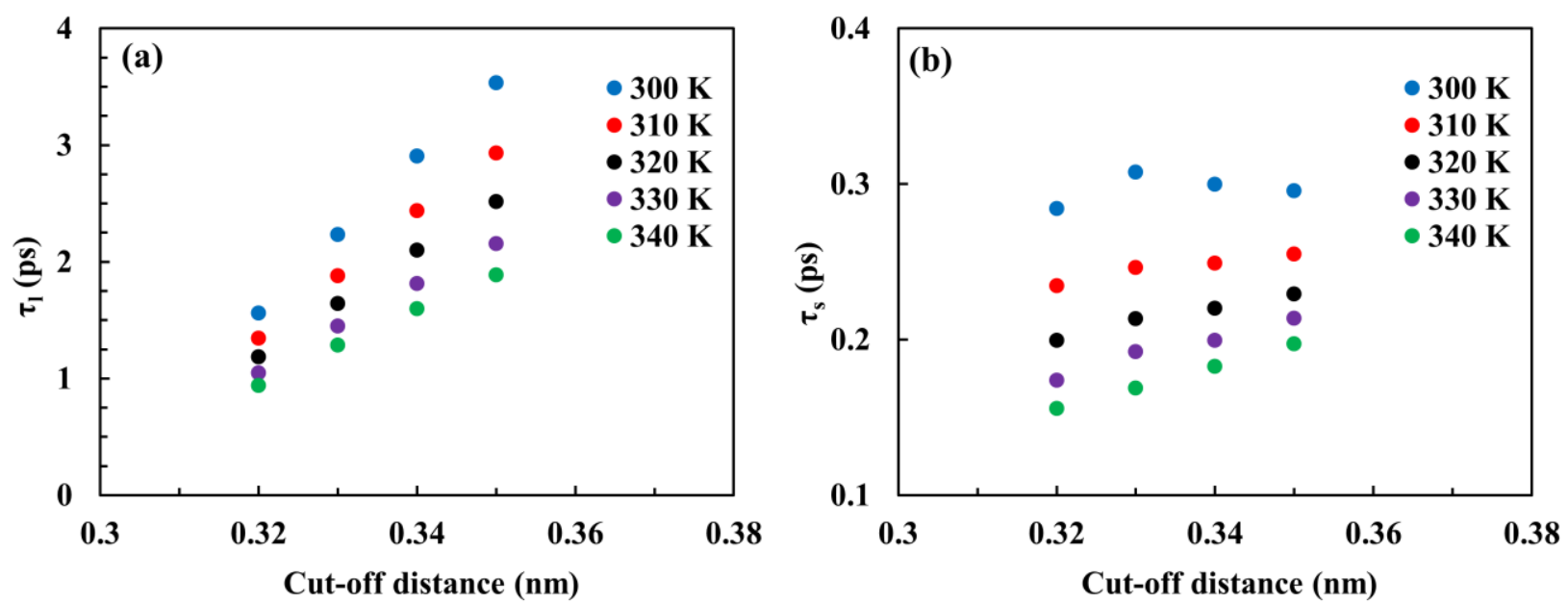

Figure S7. The residence time of water around water at varying cut-off distances. These results are based on the rigid-rod model with a rod-rod distance of $1.4 \mathrm{~nm}$. Both $\tau_{1}$ and $\tau_{\mathrm{s}}$ are dependent on the cut-off distance. This dependence emphasizes the importance of consistent cut-off distances. We would also like to mention that the linear dependence of $D$ on $1 / \tau_{1}$ stands in all the cut-off distances. 


\section{Dependence of the residence times on the density}

Table S1. With changing temperature, the density of the system is also changing, although not to a significant extent (less than 5\%). To evaluate the impact of density on residence time, we recalculate the residence time of water around water with the same density at varying temperatures. The table below shows the residence time of bulk SPC/E water with equilibrated density $\left(\tau_{\mathrm{s}}, \tau_{\mathrm{l}}\right)$ and the same density $\left(\tau_{\mathrm{s}, \mathrm{sd}}, \tau_{1, \mathrm{sd}}\right)$. To achieve constant density, we used the simulation box of an equilibrated system under $300 \mathrm{~K}$ to all the other temperatures in the NVT ensemble. The change of density with temperature appears to have little effect on the residence time, at least within this temperature range.

\begin{tabular}{ccccc}
\hline Temperature $(\mathbf{K})$ & $\boldsymbol{\tau}_{\mathbf{s}}(\mathbf{p s})$ & $\boldsymbol{\tau}_{\mathbf{s , s d}}(\mathbf{p s})$ & $\boldsymbol{\tau}_{\mathbf{l}}(\mathbf{p s})$ & $\boldsymbol{\tau}_{\mathbf{1 , s d}}(\mathbf{p s})$ \\
\hline 260 & 0.44 & 0.42 & 3.88 & 3.76 \\
270 & 0.30 & 0.31 & 3.03 & 3.00 \\
280 & 0.25 & 0.26 & 2.50 & 2.52 \\
290 & 0.21 & 0.21 & 2.12 & 2.13 \\
300 & 0.17 & 0.17 & 1.78 & 1.78 \\
310 & 0.16 & 0.15 & 1.56 & 1.56 \\
320 & 0.14 & 0.15 & 1.38 & 1.39 \\
330 & 0.14 & 0.14 & 1.23 & 1.23 \\
340 & 0.14 & 0.13 & 1.07 & 1.12 \\
\hline
\end{tabular}


8. Calculations of residence times with TIP4P/water model.
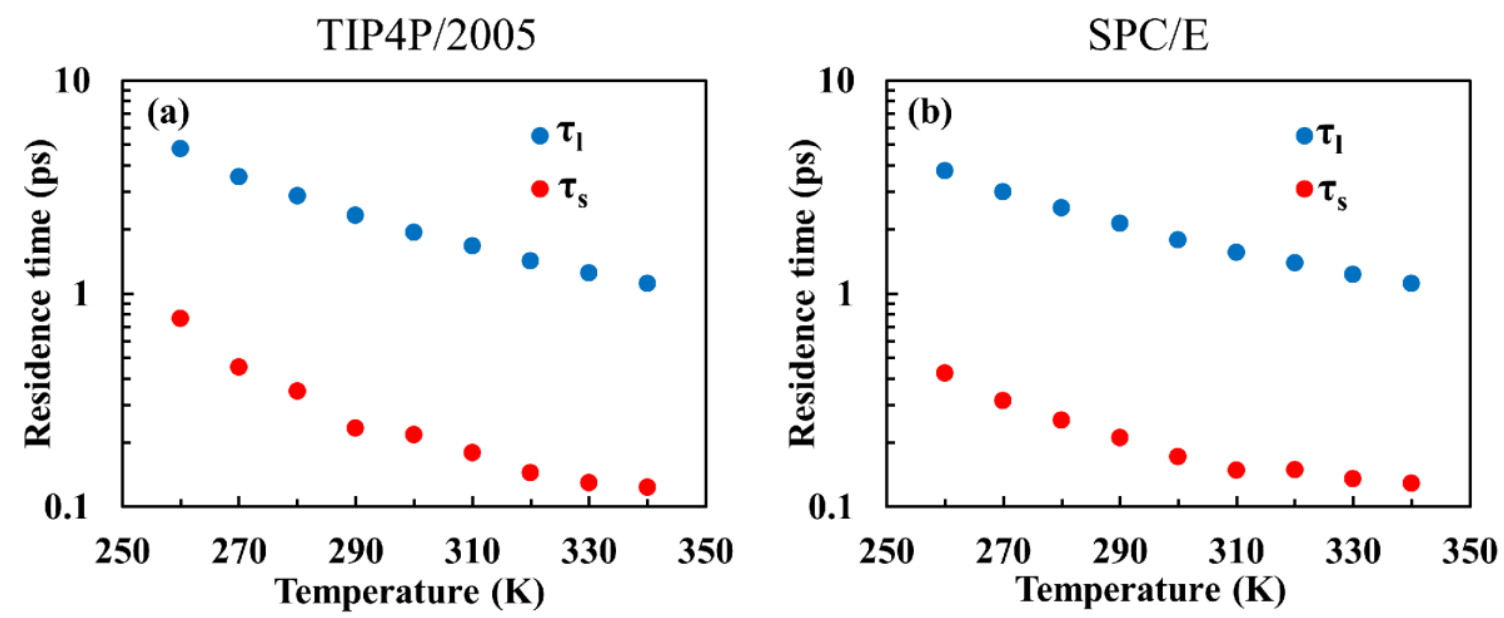

Figure S8. Comparing residence time of water around water between (b) bulk SPC/E water and (a) bulk TIP4P/2005 water. The TIP4P/2005 water shows slightly higher residence time than SPC/E water, consistent with the lower diffusion coefficients predicted by the TIP4P/2005 water model. 

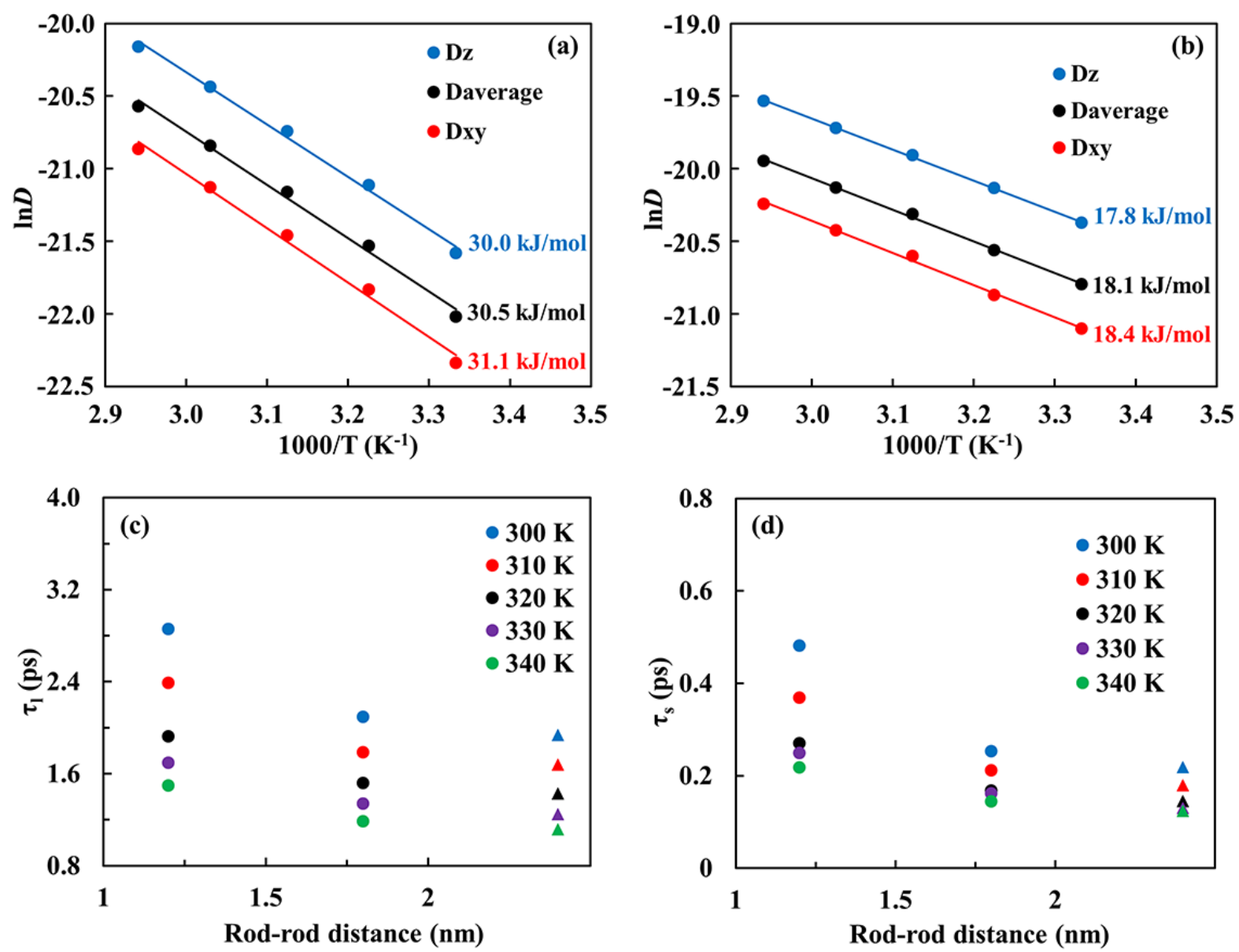

Figure S9. Panel (a) and (b) show the Arrhenius plots of confined TIP4P/2005 water in the rigidrod model with rod-rod distances of $1.2 \mathrm{~nm}$ and $1.8 \mathrm{~nm}$. The temperature range is between $300 \mathrm{~K}$ - $340 \mathrm{~K}$ with a $10 \mathrm{~K}$ interval. $E_{a}$ of bulk TIP4P/2005 water at the same temperature range is 16.2 $\mathrm{kJ} / \mathrm{mol}$. Panel (c) and (d) summarize the residence time for confined (circle) and bulk (triangle) TIP4P/2005 water. We observe similar trends as in those of confined SPC/E water. 


\section{Physical implications of $\tau_{l}$ and the correlation between $\tau_{l}$ and $D$}
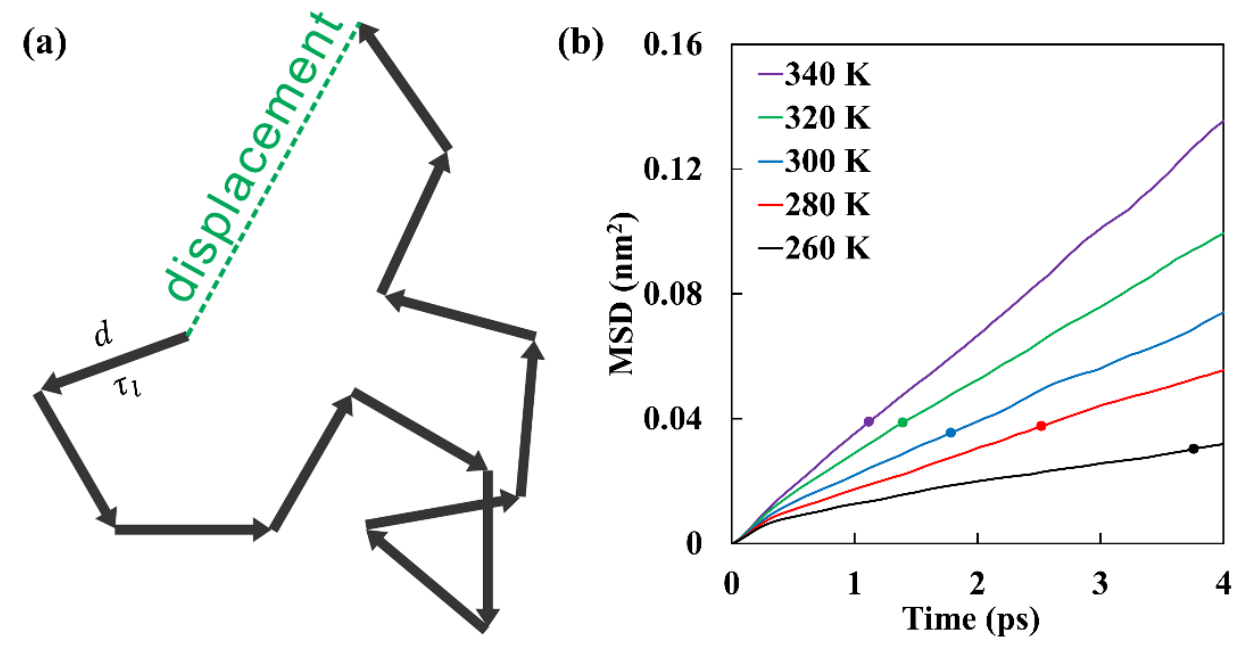

Figure S10. We start from the random walk picture to explain $\tau_{l}$ and its relation to the diffusion coefficient. The long residence time $\tau_{l}$ stands for the average time it takes for associated molecules to separate from each other. In other words, a molecule exits the vicinity of its initial neighbors at the end of $\tau_{l}$. Thus, the displacement of a molecule within $\tau_{l}$ is a random step. We discover that the displacement during $\tau_{l}$, referred to as $\mathrm{d}$ in panel (a), is not strongly dependent on the temperature when looking at the same system. Panel (b) represents the mean squared displacement (MSD) of bulk water as a function of diffusion time at varying temperatures. The bubbles on the plots represent $\tau_{l}$ at the respective temperatures. The MSD of water molecules at $\tau_{l}$ ranges between $0.034 \mathrm{~nm}^{2}$ and $0.036 \mathrm{~nm}^{2}$ at these temperatures. Thus, it takes similar displacement for water molecules to exit their immediate solvation shell under varying temperatures. Assuming there are $n$ random steps, the total diffusion time equals to $n \tau_{l}$, and the MSD equals $6 D n \tau_{l}$ (threedimensional diffusion). We can assume that MSD is directly proportional to the number of random steps when $\mathrm{d}$ is a constant, providing there is enough time and number averaging. Thus, $D \tau_{l}$ is a 
constant, and $D$ is proportional to $1 / \tau_{l}$. The calculation of residence times supports that $D$ is linear with $1 / \tau_{l}$ with a small deviation from the proportional relationship. 
10. Residence time of water around water in the rigid-rod model at a rod-rod distance of 1.8 nm with modified hydrophobicity
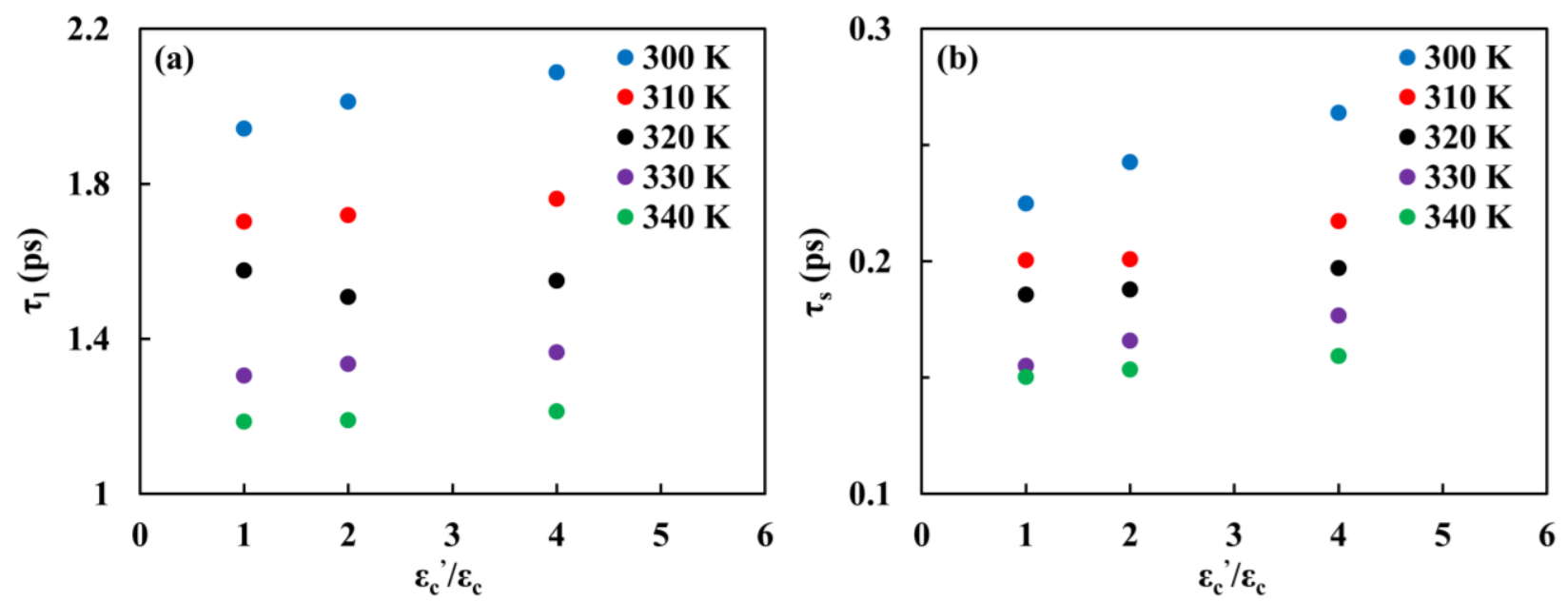

Figure S11. The residence time of water around water as a function of the hydrophobicity of the rigid rods at a rod-rod distance of $1.8 \mathrm{~nm}$. Overall, both $\tau_{l}$ and $\tau_{s}$ increase with increasing watersurface interactions. 


\section{Residence time of water in the first surface layer and further layers}

Table S2. Long residence time $\tau_{l}$ of water in the rigid-rod model as a function of cut-off distance. The $0-0.55 \mathrm{~nm}$ range corresponds to the first surface layer while the other ranges incorporate further water layers.

\begin{tabular}{ccc}
\hline Distance to center of rods $(\mathrm{nm})$ & \multicolumn{2}{c}{$\tau_{l}(\mathrm{ps})$} \\
\hline & Original force field & $\varepsilon_{\mathrm{c}}{ }^{\prime} / \varepsilon_{\mathrm{c}}=2$ \\
$0-0.55$ & 2.33 & 2.72 \\
$0.55-0.7$ & 2.31 & 2.61 \\
$0.7-0.9$ & 2.13 & 2.21 \\
All & 1.94 & 2.09 \\
\hline
\end{tabular}


12. Impact of time interval on the calculation of residence time correlation function $S(t)$

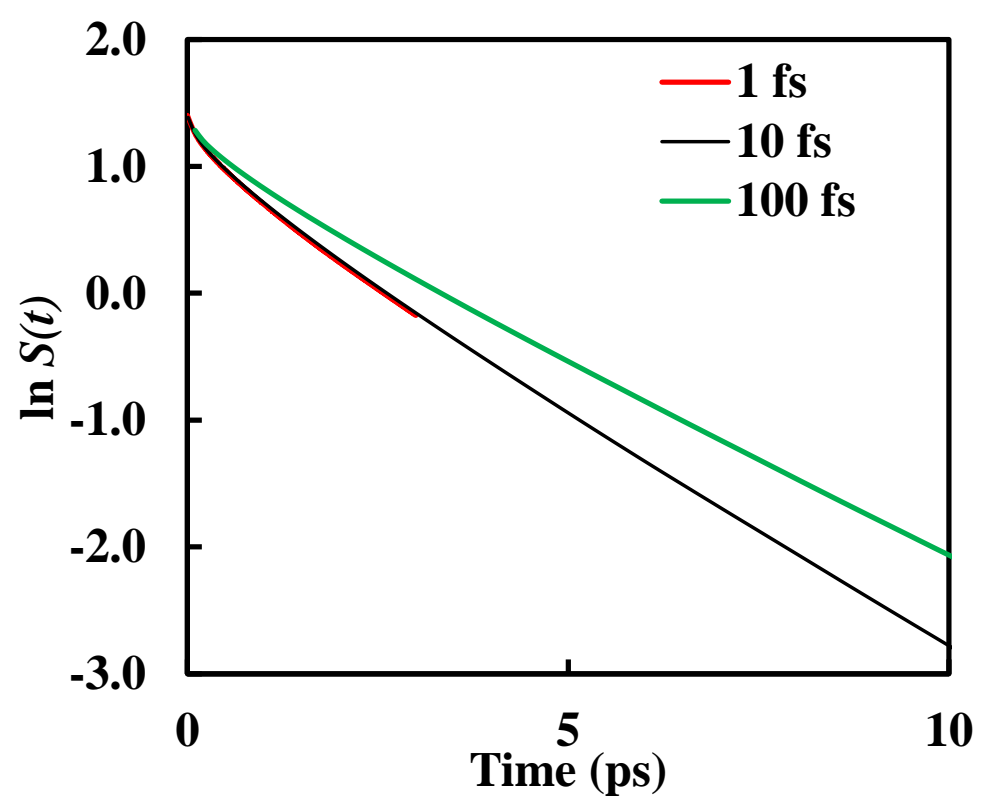

Figure S12. Plots of time correlation function $S(t)$ calculated using three different time intervals: $1 \mathrm{fs}, 10 \mathrm{fs}$, and $100 \mathrm{fs}$. The simulation system selected is the rigid-rod model with a rod-rod distance of $1.2 \mathrm{~nm}$ at $300 \mathrm{~K}$. With a longer time interval, the calculation overestimates the correlation time between molecules, thus leading to a slower-decaying $S(t)$. We notice that $S(t)$ is very similar between $1 \mathrm{fs}$ time interval and $10 \mathrm{fs}$ time interval, while the $1 \mathrm{fs}$ time interval requires a factor of 10 longer computational time. A $10 \mathrm{fs}$ interval is therefore both accurate and computationally feasible. 
13. Pre-diffusive behavior in simulation models

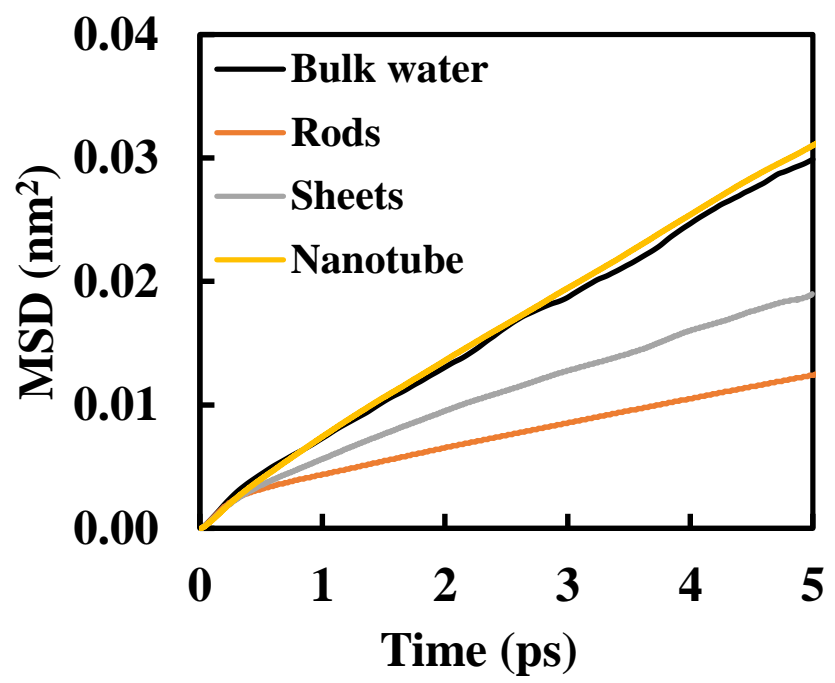

Figure S13. Picosecond timescale MSD plots of water in bulk and confined systems (rigid-rod model with $1.2 \mathrm{~nm}$ rod-rod distance, parallel sheets model with $0.7 \mathrm{~nm}$ separation, and nanotube model with $1.6 \mathrm{~nm}$ tube diameter). A steeper-sloped pre-diffusive regime is observed during the starting 1 ps period for all simulation models. The shallower sloped diffusive regime occurs after this pre-diffusive regime. During the diffusive regime, the MSD is clearly linear with diffusion time.

\section{References}

1. Kohler, M. H.; Bordin, J. R.; da Silva, L. B.; Barbosa, M. C., Breakdown of the Stokes-Einstein water transport through narrow hydrophobic nanotubes. Phys. Chem. Chem. Phys. 2017, 19, 1292112927.

2. Sadeghi, M.; Parsafar, G. A., Density-induced molecular arrangements of water inside carbon nanotubes. Phys. Chem. Chem. Phys. 2013, 15, 7379-7388. 